\title{
The prevalence of coeliac disease-associated human leukocyte antigens in South African transplant donors and recipients
}

\author{
K L Mrubata, ${ }^{1}$ MB ChB, DCH (SA), FC Paed (SA), MMed (Paed); P Barrow, ${ }^{2}$ MB BCh, DCH, FCP, Cert Gastroenterol (SA); \\ E Mayne, ${ }^{3}$ BA, MB BCh, MMed (Haem), FC Path (SA) Haem; D Nelson, ${ }^{4}$ Dip Biomedical Technology; \\ T De Maayer, ${ }^{1}$ MB BCh, FC Paed, MMed (Paed), Cert Gastroenterology (SA) Paed

\begin{abstract}
${ }^{1}$ Department of Paediatrics, Rahima Moosa Mother and Child Hospital and Department of Paediatrics and Child Health, School of Clinical Medicine, Faculty of Health Sciences, University of the Witwatersrand, Johannesburg, South Africa

${ }^{2}$ Wits Donald Gordon Medical Centre, Johannesburg, South Africa

${ }^{3}$ Department of Immunology, School of Pathology, Faculty of Health Sciences, University of the Witwatersrand and National Health Laboratory Service, Johannesburg, South Africa
\end{abstract} \\ ${ }^{4}$ Specialised Laboratory Service, South African National Blood Service, Johannesburg, South Africa
}

Corresponding author: T De Maayer (tim.demaayer@wits.ac.za)

\begin{abstract}
Background. Coeliac disease (CD) is an autoimmune condition occurring in genetically predisposed individuals exposed to an environmental trigger. The human leukocyte antigen (HLA) haplotypes HLA-DQ2.5 and HLA-DQ8 have the strongest association with CD, and $90-95 \%$ of CD patients bear these haplotypes. The susceptibility of the South African (SA) population to CD has not been studied previously.

Objectives. To describe the genetic propensity of the SA population to CD.

Methods. The South African National Blood Service database was used to analyse the prevalence of HLA-DQ2.5 and HLA-DQ8 in potential donors and recipients of organ transplants. Self-reported ethnic group was used to estimate the prevalence among different population groups.

Results. The overall prevalence of $H L A-D Q 2.5$ and $H L A-D Q 8$ was $19.8 \%$. The prevalence was lower in black participants (15.9\%) than in whites $(28.6 \%)$. Coloured $(22.0 \%)$ and Indian (17.4\%) participants had an intermediate prevalence. There was no significant difference between potential transplant donors and recipients.

Conclusions. The prevalence of HLA-DQ2.5 and HLA-DQ8 differed among SA study participants of different ethnicities. However, the notion that $\mathrm{CD}$ does not occur in black South Africans owing to lack of a genetic predisposition is incorrect.

S Afr Med J 2021;111(10):991-994. https://doi.org/10.7196/SAMJ.2021.v111i10.15680
\end{abstract}

Coeliac disease $(\mathrm{CD})$ was previously thought to be a disease of people of European descent, but this perception has changed in the past few decades. ${ }^{[1]}$ More recently, CD has been described to be prevalent in the Middle East, South America and North Africa. ${ }^{[1]}$ There is a notable lack of studies on CD in sub-Saharan Africa, and the perception that $\mathrm{CD}$ does not exist in black southern African patients persists. The disease is therefore often not often considered in patients with compatible clinical presentations such as chronic diarrhoea and malnutrition, two common conditions in South Africa (SA). ${ }^{[2]}$ Furthermore, atypical presentations of CD (e.g. iron deficiency, altered bone metabolism, elevated serum liver enzymes, short stature) have been shown to be common, leaving much of the CD 'iceberg' unrecognised. ${ }^{[3]}$

The genetic system bearing the strongest disease association with $\mathrm{CD}$ is the human leukocyte antigen (HLA) major histocompatibility class II genes HLA-DQ2.5 and HLA-DQ8. ${ }^{[4]}$ Approximately 90 - 95\% of CD patients carry HLA haplotype HLA-DQ2.5 heterodimers (encoded by $D Q A 1^{*} 05$ and $D Q B 1^{*} 02$ alleles) or $H L A-D Q 8$ $\left(D Q B 1^{*} 03: 02\right)$ in combination with a $D Q A 1^{*} 03$ variant. ${ }^{[5]}$ The remaining $5 \%$ of $\mathrm{CD}$ patients without $H L A-D Q 2.5$ or HLA-DQ8 are thought to have an $H L A-D Q 2.2$ haplotype (DQA1*02:01$\left.D Q B 1{ }^{*} 02: 02\right){ }^{[6]}$ Although several genomic areas outside the HLA region associated with $C D$ have been identified, their contribution to predisposition to $\mathrm{CD}$ is relatively small. ${ }^{[5]}$

\section{Objectives}

In southern Africa, the general population's predisposition to $\mathrm{CD}$ has not been reported. The objective of this retrospective study was to report on the prevalence of HLA-DQ2.5 and HLA-DQ8 in the SA population using HLA data collected by the Tissue Immunology Laboratory at the South African National Blood Service (SANBS) for the purposes of transplantation.

\section{Methods}

Ethical approval was obtained from the University of the Witwatersrand Human Research Ethics Committee (ref. no. M190670) and the SANBS Human Research Ethics Committee (ref. no. 2019/0481). All patients typed as part of the tissue immunology work-up or for inclusion on the bone marrow registry (through the Sunflower Fund) were included. HLA typing was performed by lowto medium-resolution Luminex typing utilising a Labtype rSSO kit (One Lambda, Thermo Fisher Scientific, USA) and acquired on LabScan 200 and Flex Map 3D (Thermo Fisher Scientific, USA). Briefly, this process utilises bead-complexed sequence-specific oligonucleotides to define genetic HLA typing. HLA class II data were expressed in a two-digit format. Data were recorded on the SANBS electronic database and extracted in a de-identified format. Where known, patient ethnicity, gender and age and reason for typing were also included. Ethnicity was self-reported and classified 
as per Statistics South Africa into one of four categories: black, white, coloured and Indian. ${ }^{[7]}$

The presence or absence of $H L A-D Q 2.5$ (encoded $D Q B 1{ }^{*} 02$ $\left.D Q A 1^{*} 05\right), H L A-D Q 8\left(D Q B 1^{*} 03: 02 D Q A 1^{*} 03\right)$ and $H L A-D Q 2.2$ $\left(D Q A 1^{\star} 02: 01-D Q B 1^{\star} 02: 02\right)$ haplotypes was determined. Participant HLA results were divided into three risk groups according to their HLA class II status: high risk when the participant was homozygous for HLA-DQ2.5 or bore both HLA-DQ8 and HLA-DQ2.5 haplotypes, moderate risk when the participant haplotypes were heterozygous for $H L A-D Q 2.5$ or $H L A-D Q 8$, and low risk with $H L A-D Q 2.2$ haplotypes.

Data were analysed using Stata Intercooled version 11 (StataCorp, USA). Where appropriate, descriptive statistics were computed, including mean and standard deviation for parametric and median and interquartile range (IQR) for non-parametric continuous data. Prevalence is reported with $95 \%$ confidence intervals (CIs), and $\chi^{2}$ statistics were used to compare prevalences in different groups. A $p$-value $<0.05$ was considered significant.

\section{Results}

The records of 5746 persons who underwent HLA typing were included. The median (IQR) age of the 5383 participants whose age was known was 36 (24 - 48) years (range 0 - 91 years). The most common reason for HLA testing was chronic renal failure requiring kidney transplantation (37.7\%), while $28.3 \%$ were tested as potential organ donors; $17.9 \%$ did not have the reason for testing recorded. Further general population characteristics are set out in Table 1.

In total, 1135 participants (19.8\%; 95\% CI 18.7 - 20.8) were positive for $H L A-D Q 2.5$ or $H L A-D Q 8$. Blacks had a significantly lower prevalence (15.9\%; 95\% CI 14.3 - 17.5) than whites (28.6\%; 95\% CI 25.5 - 31.8 (OR 2.11; 95\% CI $1.74-2.56$ ); $p<0.001$ ). Coloureds (22.0\%; 95\% CI 11.1 - 32.9), Indians (17.4\%; 95\% CI 14.6 - 20.2) and those of unspecified race $(20.8 \%$; $95 \%$ CI $19-22.4)$ had an intermediate prevalence of the HLA-DQ2.5 or HLA-DQ8 haplotypes (Table 1). There were no differences observed between the age and gender in the $H L A-D Q 2.5-$ or $H L A-D Q 8$-positive v. negative groups (median age 36 years in both groups, female gender in $43.5 \%$ v. $41.7 \%$, respectively).

There was no difference in the prevalence of HLA-DQ2.5 or HLA$D Q 8$ positivity between potential organ donors v. recipients $(20.2 \% \mathrm{v}$. $20.0 \%$; $p=0.96$ ).

Of the study subjects, $2.2 \%(n=124)$ were homozygous for HLA$D Q 2.5$ or had both HLA-DQ2.5 and HLA-DQ8 genotypes (high risk, Table 2). This group included 35 (1.7\%) black, 1 (1.7\%) coloured, $12(1.7 \%)$ Indian and $26(3.3 \%)$ white participants. The HLA-DQ2.2 haplotype, which confers a low risk of $\mathrm{CD}$, was more prevalent among Indians at $15.2 \%$ (low risk, Table 2).

\section{Discussion}

This retrospective audit sought to determine the prevalence of $\mathrm{CD}$-associated haplotypes using the HLA information from potential transplant donors and recipients in the SANBS database. We observed that the prevalence of $H L A-D Q 2.5$ and $D Q 8$ haplotypes in this study sample was almost $20 \%$, which is lower than reported in studies performed in many Western countries, which have a prevalence of $\sim 30 \%{ }^{[5]}$

CD-associated HLA haplotypes were identified across all ethnic groups. Significant differences between ethnicities were observed,

\begin{tabular}{|c|c|c|c|}
\hline Demographic categories & Total $(N=5746), n$ & $H L A-D Q 2.5, n(\%)$ & $H L A-D Q 2.5$ or $H L A-D Q 8, n(\%)$ \\
\hline \multicolumn{4}{|l|}{ Gender } \\
\hline Female & 2417 & $476(19.7)$ & $494(20.4)$ \\
\hline Male & 3070 & $564(18.4)$ & $588(19.2)$ \\
\hline Unspecified & 259 & 49 (18.9) & $53(20.5)$ \\
\hline \multicolumn{4}{|l|}{ Age group (years) } \\
\hline $0-17$ & 836 & $135(16.1)$ & $141(16.9)$ \\
\hline $18-35$ & 1778 & $365(20.5)$ & $380(21.4)$ \\
\hline $36-53$ & 2003 & $378(18.9)$ & $395(19.7)$ \\
\hline $54-71$ & 753 & $147(19.5)$ & $151(20.1)$ \\
\hline$\geq 72$ & 13 & $3(23.1)$ & $3(23.1)$ \\
\hline Unspecified & 363 & $61(16.8)$ & $65(17.9)$ \\
\hline \multicolumn{4}{|l|}{ Ethnicity } \\
\hline Black & 2034 & $321(15.8)$ & $324(15.9)^{*}$ \\
\hline White & 800 & $216(27.0)$ & $229(28.6)^{*}$ \\
\hline Indian & 690 & $113(16.4)$ & $120(17.4)$ \\
\hline Coloured & 59 & $11(18.6)$ & $13(22.0)$ \\
\hline Unspecified & 2163 & $428(19.8)$ & $449(20.8)$ \\
\hline \multicolumn{4}{|l|}{ Reason for HLA testing } \\
\hline Chronic renal failure & 2165 & $446(20.6)$ & $451(20.8)$ \\
\hline Donor & 1623 & $302(18.6)$ & $327(20.1)$ \\
\hline Leukaemia & 398 & $68(17.1)$ & $70(17.6)$ \\
\hline Aplastic anaemia & 118 & $19(16.1)$ & $19(16.1)$ \\
\hline Cardiac transplant & 100 & $25(25.0)$ & $25(25.0)$ \\
\hline Lung transplant & 45 & $9(20.0)$ & $11(24.4)$ \\
\hline Other & 269 & $39(14.5)$ & 45 (16.7) \\
\hline Not specified & 1028 & $181(17.6)$ & $187(18.2)$ \\
\hline
\end{tabular}


Table 2. Coeliac disease risk categories stratified by ethnicity

\begin{tabular}{llll}
\hline Ethnicity & High risk, $\boldsymbol{n}(\%)$ & Moderate risk, $\boldsymbol{n}(\%)$ & Low risk, $\boldsymbol{n}(\%)$ \\
\hline Black $(N=2$ 034) & $35(1.7)$ & $324(15.9)$ & $191(9.4)$ \\
White $(N=800)$ & $26(3.3)$ & $229(28.6))$ & $91(11.4)$ \\
Indian $(N=690)$ & $12(1.7)$ & $120(17.4)$ & $105(15.2)$ \\
Coloured $(N=59)$ & $1(1.7)$ & $13(22.0)$ & $3(5.1)$ \\
Unspecified $(N=2$ 163) & $50(2.3)$ & $449(20.8)$ & $255(11.8)$ \\
Total $(N=5746)$ & $124(2.2)$ & $1135(19.8)$ & $645(11.2)$ \\
Percentages calculated as percentage of row total. \\
High risk = homozygous for HLA-DQ2.5 or HLA-DQ2.5 and HLA-DQ8-positive; Moderate risk = HLA-DQ2.5- or HLA-DQ8-positive; Low risk = HLA-DQ2.2.
\end{tabular}

with higher prevalences in white and coloured participants, at $28.6 \%$ and $22.0 \%$, respectively, and a prevalence of $15.9 \%$ among black participants. This finding would suggest that all groups are susceptible to CD.

An HLA-associated predisposition is not sufficient to cause CD, as demonstrated in prospective studies by Liu et al. ${ }^{\left[{ }^{[8]}\right.}$ where $3 \%$ and $11 \%$ of children with heterozygous and homozygous HLA-DQ2.5 haplotypes, respectively, developed CD by age 60 months. Dietary gluten intake is an obvious prerequisite. In $\mathrm{SA}$, maize remains the most consumed staple food, but wheat consumption is not far off the world consumption rate (60 v. $66 \mathrm{~kg} / \mathrm{capita} /$ year) ${ }^{[9]}$ The role of gluten intake during the first 5 years of life in the development of $\mathrm{CD}$ remains controversial, but a recent prospective multinational cohort study followed 6605 children who were $H L A-D Q 2.5-$ or $H L A-D Q 8$-positive. The authors conclude that gluten intake $>1 \mathrm{~g} / \mathrm{d}$ above the reference amount was associated with an increased absolute risk of CD of $7.2 \% \cdot{ }^{[10]}$ Although the gluten intake in young SA children remains undocumented, the 1999 National Food Consumption Survey ${ }^{[1]}$ revealed that bread was among the foods most commonly eaten by children aged between 1 and 9 years. However, the timing of gluten introduction to infants at risk for CD and the role of breastfeeding do not appear to have a protective effect. ${ }^{[12]}$

Further factors that may influence the development of CD include gastrointestinal infections, other genetic factors, the gut microbiome, the innate immune system, and host-microbiome interactions. ${ }^{[12,13]}$ These factors remain poorly understood, and it is unclear how they may influence the development of clinical CD in SA. However, it may be hypothesised that higher exposure to gastrointestinal infections, less antibiotic use and a different microbiome may be protecting South Africans.

Alternatively, a lack of awareness and the false belief that $\mathrm{CD}$ does not occur in black ethnic groups may lead to a lowered detection rate and therefore a low prevalence of diagnosed CD in sub-Saharan Africa. This is supported by Paruk et al., ${ }^{[14]}$ who reported a similar prevalence of CD in SA black patients with type 1 diabetes as studies from Western countries. Poor access to serological screening tests and confirmatory endoscopy may have an additional effect, as not all healthcare centres are well resourced or adequately equipped in our setting. Overall, further study is required to investigate the prevalence of CD in SA and explore the reasons affecting its prevalence or lack thereof.

\section{Study limitations}

The study relied on a sample of existing data with various indications for transplantation, some of which may overlap or have association with the extraintestinal manifestations of $\mathrm{CD}$. However, there was no difference in the CD-associated HLA haplotypes of donors and recipients. Self-reported ethnicity may not be accurate in all cases, and ethnicity was unknown in a large percentage of participants, resulting in poor representation of minority groups. It is not known whether under-reporting of ethnicity was more prevalent in any group.

\section{Conclusions and recommendations}

This study has shown that the prevalence of the $\mathrm{CD}$-associated haplotypes in potential donors and recipients of organ transplants in the SANBS database is $20 \%$. CD-associated haplotypes were more common in white participants, but were present in all population groups. We recommend that clinicians screen for $\mathrm{CD}$ using anti-tissue transglutaminase antibodies in children and adults with symptoms associated with $\mathrm{CD}$, and in those who are at an increased risk for $\mathrm{CD}$ (e.g. type 1 diabetes mellitus, autoimmune thyroid disease), regardless of ethnicity, as suggested in international guidelines. ${ }^{[15,16]}$ Positive antibody results should be confirmed with endoscopy and duodenal biopsies, at least until the true prevalence of CD in SA is better understood.

Furthermore, heightened awareness and large population-based studies are required to determine the prevalence of $\mathrm{CD}$ in southern Africa.

Declaration. The research for this study was done in partial fulfilment of the requirements for KLM's MMed (Paediatrics) degree at the University of the Witwatersrand.

Acknowledgements. The authors thank the SANBS for their assistance and data access.

Author contributions. KLM: study design, data analysis, writing of initial manuscript; DN: data retrieval and manuscript review; PB: study design and manuscript review; EM: manuscript review; TDM: study design, data analysis, manuscript review.

Funding. None.

Conflicts of interest. None.

1. Cataldo F, Montalto G. Celiac disease in the developing countries: A new and challenging public healt problem. World J Gastroenterol 2007;13(15):2153-2159. https://doi.org/10.3748/wgg.v13.i15.2153

. Reid AE, Hendricks MK, Groenewald P, Bradshaw D. Where do children die and what are the causes? Under-5 deaths in the Metro West geographical service area of the Western Cape, South Africa, 2011. S Afr Med J 2016;106(4):359-364. https://doi.org/10.7196/SAMJ.2016.v106i4.10521

3. Almallouhi $\mathrm{E}$, King KS, Patel B, et al. Increasing incidence and altered presentation in a populationbased study of pediatric celiac disease in North America. J Pediatr Gastroenterol Nutr 2017;65(4):432437. https://doi.org/10.1097/mpg.0000000000001532

4. Brown NK, Guandalini S, Semrad C, Kupfer SS. A clinician's guide to celiac disease HLA genetics. Am J 4. Brown NK, Guandalini S, Semrad C, Kupfer SS. A clinician's guide to celiac disease HLA g
Gastroenterol 2019;114(10):1587-1592. https://doi.org/10.14309/ajg.0000000000000310

Gastroenterol 2019;114(10):1587-1592. https://doi.org/10.14309/ajg.0000000000000310
Megiorni F, Pizzuti A. HLA-DQA1 and HLA-DQB1 in celiac disease predisposition: Practical Megiorni F, Pizzuti A. HLA-DQA1 and HLA-DQB1 in celiac disease predisposition: Practical
implications of the HLA molecular typing. J Biomed Sci 2012;19:88. https://doi.org/10.1186/14230127-19-88

6. Mubarak A, Spierings E, Wolters V, van Hoogstraten I, Kneepkens CM, Houwen R. Human leukocyte antigen DQ2.2 and celiac disease. J Pediatr Gastroenterol Nutr 2013;56(4):428-430. https://doi. org/10.1097/mpg.0b013e31827913f9

Khalfani AK, Zuberi T. Racial classification and the modern census in South Africa, 1911 - 1996. Race Soc 2001;4(2):161-176. https://doi.org/10.1016/S1090-9524(03)00007-X

. Liu E, Lee H-S, Aronsson CA, et al. Risk of pediatric celiac disease according to HLA haplotype and country. N Engl J Med 2014;371(1):42-49. https://doi.org/10.1056/NEJMoal313977

Ronquest-Ross LC, Vink N, Sigge GO. Food consumption changes in South Africa since 1994. S Afr J Sci 2015;111(9-10), Art. \#2014-0354. https://doi.org/10.17159/sajs.2015/20140354

10. Andrén Aronsson C, Lee H-S, Hård af Segerstad EM, et al. Association of gluten intake during the first 5 years of life with incidence of celiac disease autoimmunity and celiac disease among children at first 5 years of life with incidence of celiac disease autoimmunity and celiac disease an
increased risk. JAMA 2019;322(6):514-523. https://doi.org/10.1001/jama.2019.10329

11. Labadarios D, Steyn NP, Maunder E, et al. The National Food Consumption Survey (NFCS): South Africa, 1999. Public Health Nutr 2005;8(5):533-543. https://doi.org/10.1079/phn2005816

Africa, 1999. Public Health Nutr 2005;8(5):533-543. https://doi.org/ $10.1079 / \mathrm{phn} 2005816$
12. Lionetti E, Catassi C. The role of environmental factors in the development of celiac disease: What is new? Diseases 2015;3(4):282-293. https://doi.org/10.3390/diseases3040282 
13. Nylund L, Kaukinen K, Lindfors K. The microbiota as a component of the celiac disease and non-celiac gluten sensitivity. Clin Nutr Exp 2016;6:17-24. https://doi.org/10.1016/j.yclnex.2016.01.002

14. Paruk IM, Naidoo VG, Pirie FJ, et al. Prevalence and characteristics of celiac disease in South African patients with type 1 diabetes mellitus: Results from the Durban Diabetes and Celiac Disease Study. patients with type 1 diabetes mellitus: Results from the Durban Diabetes and

15. Husby S, Koletzko S, Korponay-Szabo I, et al. European Society Paediatric Gastroenterology, Hepatology and Nutrition Guidelines for Diagnosing Coeliac Disease 2020. J Pediatr Gastroenterol Nutr 2020;70(1):141-156. https://doi.org/10.1097/mpg.0000000000002497
16. Bai JC, Ciacci C. World Gastroenterology Organisation Global Guidelines: Celiac Disease February 2017. J Clin Gastroenterol 2017;51(9):755-768. https://doi.org/10.1097/mcg.0000000000000919

Accepted 18 May 2021 